\title{
On-demand Bandwidth and Stability Based Unicast Routing in Mobile Adhoc Networks
}

\author{
P. I. Basarkod and S. S. Manvi
}

\begin{abstract}
Characteristics of mobile ad hoc networks (MANETs) such as lack of central coordination, dynamic topology and limited resources pose a challenging problem in quality of service $(\mathrm{QoS})$ routing. Providing an efficient, robust and low overhead QoS unicast route from source to destination is a critical issue. Bandwidth and route stability are the major important QoS parameters for applications where long duration connections are required with stringent bandwidth requirements for multimedia applications. This paper proposes an On-demand Bandwidth and Stability based Unicast Routing scheme (OBSUR) in MANET by adding additional QoS features to existing Dynamic Source Routing (DSR) protocol. The objective of the OBSUR is to provide QoS satisfied, reliable and robust route for communicating nodes. The scheme works in following steps. (1) Each node in the network periodically (small regular intervals) estimates bandwidth availability, node and link stability, buffer availability, and stability factor between nodes. (2) Construction of neighbor stability and QoS database at every node which is used in route establishment process. (3) The unicast path is constructed by using route request and route reply packets with the help of route information cache, and (4) route maintenance in case of node mobility and route failures. Simulation results show that there is an improvement in terms of traffic admission ratio, control overhead, packet delivery ratio, end to end delay and throughput as compared to Route Stability Based QoS Routing (RSQR) in MANETs.
\end{abstract}

Keywords-Mobile Ad hoc Network, QoS, stability, routing, mobility

\section{INTRODUCTION}

A MOBILE AD HOC NETWORK (MANET) consists of a collection of mobile nodes forming a dynamic autonomous network. Nodes communicate with each other without the intervention of centralized access points or base stations. In such a network, each node acts as a host, and may act as a router. Due to the limited transmission range of wireless network interfaces, multiple hops may be needed to exchange data between nodes in the network. Due to frequent changes in the network topology and limited network resources, routing in MANET experiences link failure more often.

Applications of MANETs can be found in situations such as emergency search-and-rescue operations, meetings or conventions (in which users wish to quickly share information), and data acquisition operations in hostile terrain. In situations like battlefields or major disaster areas, ad hoc networks need to be deployed immediately without base stations or wired

P. I. Basarkod and S. S. Manvi are with the Wireless Information Systems Research Lab, Department of Electronics and Communication Engineering, Reva Institute of Technology and Management, Bangalore-560064, INDIA (e-mails: \{basarkod; sunil.manvi\}@ revainstitution.org). infrastructure. These networks are typically characterized by scarce resources (bandwidth, power, etc.), lack of established backbone infrastructure, high error rates, and a dynamic topology [1].

Applications involving real time data transfer require better quality of service (QoS) for providing uninterrupted communication. The parameters that are used in QoS routing are bandwidth, node/link stability, delays, jitters and losses, and degree of connectivity. Sometimes degree of connectivity alone is used to describe QoS that is used as the criteria for preferred neighbor election [2]. Degree of connectivity alone will not characterize the link failure. Link failure may also arise from node mobility and lack of network resources as well.

Bandwidth must be satisfied in a given route for an application so that data is transferred smoothly and available for presentation at the destination even under the conditions of mobility and limited resources. For long duration connections, nodes/links on the path must be stable so that connection failures are overcome; this facilitates data transfer without interruption. Link stability indicates how long a link can support communications between two nodes? Stability of links can be estimated by using many parameters like - signal strength, pilot signals, relative speed between two nodes forming the link, link duration distributions and remaining battery power of the nodes, etc.

The stability and lifetime of a route is determined by the number of neighbors and their connectivity, number of links in the route, stability and life time of each link in the route. The probability of route failure can be reduced by reducing the link failure rate and the number of links that compose the route. It is important to note that delay constrained routes avoid selecting very long routes.

The objective of this work is to design and analyze a unicast based on-demand routing scheme in MANET which is modified version of DSR, that provides bandwidth satisfied, reliable and robust route. The work uses node stability, link stability, buffer levels, and available bandwidth as parameters for route discovery. Stable paths are found based on selection of stable forwarding nodes that have stable connectivity and satisfy bandwidth requirement. Such a routing scheme may be suitable for applications where long duration connections are required with better link/node stability and bandwidth requirement. The designed scheme exhibits reduced control and computation overheads, reduced packet delays and enhanced packet delivery ratio. Simulation results show that there is an improvement in terms of traffic admission ratio, control overhead, packet delivery ratio, end to end delay and 
throughput as compared to Route Stability Based QoS Routing (RSQR) in MANETs.

\section{A. Related Work}

With the rapid development of multimedia applications in mobile ad hoc networks, there is an increasing need for QoS guarantee. Therefore, protocols designed for MANETs should involve satisfying application requirements while optimizing network resources. While designing routing protocols, finding of stability of nodes play an important role in order to establish a stable path. A stable path offers better packet delivery ratio and low latency. Some of the related works are as follows. In [3], randomized distributed algorithm approach is used that guarantees stablity even under high adversarial churn (nodes joining/leaving) in a poly-logarithmic number of rounds.

In [4], the stable paths are found basing on selection of stable forwarding nodes that have high stability of link connectivity. The link stability is computed by using the parameters such as received power, distance between neighboring nodes, and the link quality (bit errors in a packet). The work given in [5] introduces a QoS aware on-demand routing protocol that uses signal stability as the routing criteria along with other QoS metrics. It proposes QoS Aware Stable path Routing (QASR) designed over Signal Stability based Adaptive routing (SSA) and aims to select stable QoS routes that can survive for longer period of time.

The work given in [6] introduces a stability property called T-interval connectivity, which stipulates that for every $\mathrm{T}$ consecutive round there exists a stable connected spanning subgraph. In [7], a method is proposed that has been advocated to improve routing efficiency to select the most stable path so as to reduce the latency and the overhead. Clearly, the probabilities of path duration and path availability strongly depend on the mobility pattern of the nodes which in turn depends on the movement of a node with respect to others in the network.

The work presented in [8] reports on heuristics in order to classify nodes whose links are more stable than others. The heuristics described rely solely on local topological information and are fully distributed. Clustering techniques create hierarchical network structures, called clusters. Neighboring devices elect one appropriate device as cluster head. Due to the dynamic environment, cluster head selection becomes an important issue. The work presented in [9] analyzes synchronous as well as asynchronous heuristics for discovering nodes with prolonged topological stability. These nodes appear more appropriate to be elected as cluster heads, since the frequency of cluster head re-election and re-clustering can be decreased. The heuristics described rely on 2-hop topological information and avoid any use of geographical data.

The node location information has recently found use in solving many existing problems in MANETs. The directly communicable nodes of any node (i.e., the neighbors) and, ideally, the location of the other nodes should be available in advance to the node. In [10], authors propose a node stability-based location updating approach for which following parameters, stability of one node with respect to another node, the relative velocity of the two nodes, and battery backup of node are considered.

The beacon-less routing protocol (BLR) [11] is a positionbased routing protocol which uses the geographical location information to minimize routing overhead. BLR does not require nodes to periodically exchange beacon packets which minimizes the usage of battery power and interferences for the regular data transmission. In [12], authors propose an orientation heuristic factor to the conventional ant colony algorithm, which helps in reducing path searching time. The path finding algorithm not only makes use of the previous search findings, but also reduces the misguiding effect of pheromones on the ambiguous paths, thus reduces the problem of slow convergence.

To support energy-efficient routing, accurate state information about energy levels should be available. But due to bandwidth constraints, communication costs, high loss rate and the dynamic topology of MANETs, collecting and maintaining up-to-date state information is a very complex task. In the work given in [13], authors propose Optimized Link State Routing (OLSR) as the under-lying routing protocol and explore the accuracy of state information under different traffic rates. Energy level is taken as QoS metric, which has been used for routing decisions in many energy efficient routing protocol proposals. If nodes learn other nodes' energy level through protocol messages, fewer packets tend to get delivered in an energy-constrained network, in particular under high traffic loads or in mobile networks.

QoS based routing over MANET requires an adaptive and fast solution to path search problems. Swarm Intelligence is a machine learning technique, where intelligence can be derived from the collective behavior of natural agents. In [14], the implementation of protocol HMQAnt (Hybrid Multipath QoS Ant) is done with ACO (Ant Colony Optimization) based hybrid adhoc routing strategy for a hierarchical MANET architecture. The designed protocol gives optimum solution for adaptive and dynamically changing networks.

Multi-path routing protocols for MANET are proved to be superior over conventional single-path routing protocols since the former reduce end-to-end delay, increase reliability and provide robustness. However, the shortest path routes resulting from shortest multi-path routing, such as Ad hoc On-demand Multipath Distance Vector (AOMDV), cause the area congestion, hence its effectiveness is decreased. The work given in [15] proposes a novel AOMDV with Sufficient Bandwidth Aware (AOMDV+SBA) routing protocol which significantly improves the performance of the original AOMDV routing protocol by discovering better routes to avoid congestion.

In [16], authors propose a scheme that estimates how the node mobility effects the results on the performance of available routing strategies (i.e., path-based) and creates the framework that exploits the usually different mobility rates of the nodes by adapting the routing strategy during execution. It uses a metric for the relative mobility of the nodes, according to which the nodes are classified into mobility classes. These mobility classes determine, for any pair of origin and destination, the routing technique that best corresponds to 
their mobility properties. Moreover, special care is taken for nodes remaining almost stationary or moving with high (relative) speeds.

One critical issue for routing in MANETs is how to select a reliable path that can last longer because mobility may cause frequent breakdown in radio links. A routing mechanism based on link lifetime estimation is proposed in [17]. In this mechanism, the node's received signal strength is obtained continuously by using Newton interpolation polynomial, and by method of middle value and interception. The reference points are selected to estimate the link lifetime. With the constraint of link lifetime and hop counts, the source nodes set up the route hop by hop.

In wireless networks, context awareness and intelligence are capabilities that enable each host to observe, learn, and respond to its complex and dynamic operating environment in an efficient manner. These capabilities contrast with traditional approaches where each host adheres to a predefined set of rules, and responds accordingly. In [18], the authors propose the use of reinforcement learning (RL) to achieve context awareness and intelligence, and also presents an overview of classical RL and three extensions, including events, rules and agent interaction and coordination, to wireless networks.

The work described in [19] proposes a unicast routing protocol to effectively minimize the stability-hop count in MANETs and thereby incur lower end-to-end delay per data packet. The protocol, referred to as SILET, uses the predicted link expiration times (LETs) as part of the link weights. The weight assigned to a link is ' 1 ' plus the inverse of the LET of the link. Bandwidth Delay product based multicast routing scheme using reliable ring mesh constructed with reliability pair nodes is given in [20], where reliability is computed based on remaining battery power, differential signal strength and distance between the nodes.

In [21], the authors propose a Route Stability based QoS Routing (RSQR) protocol in MANETs which is an extension of QoS routing with throughput and delay constraints. To ensure a data path to be valid for sufficiently longer period of time, a simple model is used to compute link stability and route stability based on received signal strengths. By including some extra fields in route request/reply packets, the route stability information is utilized to select a route with higher stability among all the feasible routes between a given source destination pair. However, the work does not take in to account stability of the nodes with respect to mobility and failures.

In [22], QoS routing protocol based on alternative path selection is described. Using a combination of source routing and shortest path routing, packets are routed along alternate paths when available resources along the shortest path cannot satisfy the QoS requirements of end to end flows. Work presented in [23] deals with the processing complexity of determining QoS paths in link state based routing architectures. Authors first characterize the processing cost of QoS routing algorithms that use the widest-shortest path heuristic, then study alternatives to on-demand path computation that can reduce processing overhead. In [24], variational principles from theoretical physics are used to describe the process of routing in computer networks as an alternate approach to the traditional graph theory principles.

\section{B. Our Contributions}

In this paper, we propose an On-demand Bandwidth and Stability based Unicast Routing scheme (OBSUR) which includes the following contributions compared to existing works. (1) Designing a scheme for selecting stable nodes based on node's own stability, i.e self stability, neighbor nodes stability, buffer level and link stability. (2) Finding the stability factor based on buffer size, node's and link stability values. (3) Estimation of bandwidth at each node. (4) Maintaining up to date database at every selected stable node about stability factor and bandwidth information. (5) Maintaining Route Information Cache (RIC) to store the latest routes to destinations through Route-Request (RR) and Route-Reply (RP) packets. (6) Developing route discovery process which includes Request phase to find routes to destination using stable and QoS intermediate nodes, Reply phase to update (RIC) and confirm the routes found in request phase, and (7) designing route maintenance procedure to handle node and link failures.

The scheme works in following steps. (1) Each node in the network periodically (small regular intervals) estimates bandwidth availability, node and link stability, buffer availability, and Stability-Factor-Between-Nodes. (2) construction of neighbor stability and QoS database at every node which is used in route establishment process. (3) The unicast path is constructed by using route request and route reply packets with the help of route information cache, and (4) route maintenance in case of node mobility and route failures.

Simulation results show that there is an improvement in terms of traffic admission ratio, control overhead, packet delivery ratio, end to end delay and throughput as compared to Route Stability Based QoS Routing (RSQR) [21] in MANETs. The reason for choosing RSQR for comparison are as follows: (1) it uses QoS metrics delay and bandwidth for route stability, and (2) protocol is based on AODV (Adhoc On-demand Distance Vector routing protocol).

The rest of the paper is organized as follows. Section II discusses the proposed work in detail. Simulation and result analysis are presented in section III and conclusions are given in section IV.

\section{PRoposed WORK}

This section presents the network environment, QoS metrics including node stability and bandwidth, route establishment, route discovery and maintenance.

\section{A. Network Environment}

Figure 1 shows MANET scenario in which many heterogeneous nodes are connected. The nodes are geographically distributed in the bounded area. The nodes may randomly move in any direction with some speed in a bounded area. Each node has certain transmission range. More than one neighbor node may come under the transmission range of 


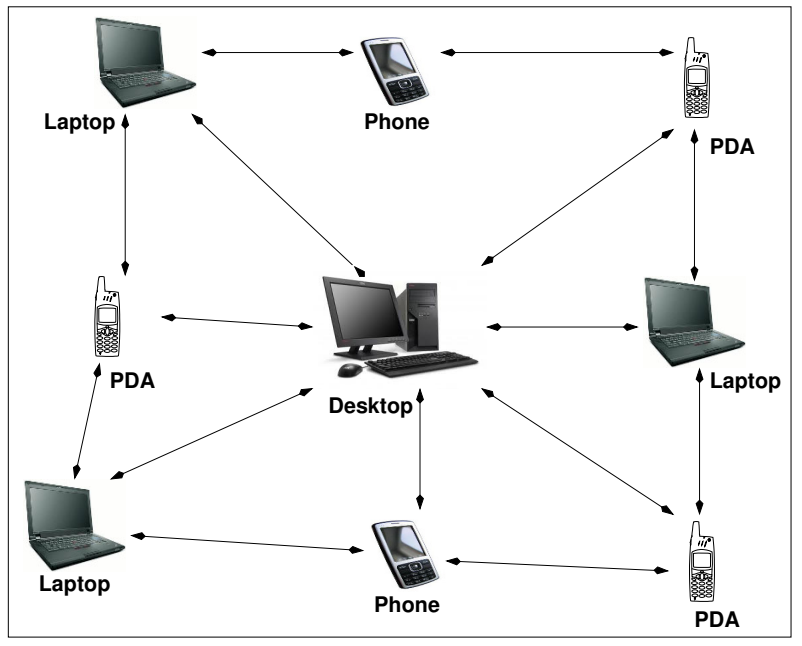

Fig. 1. Typical MANET Scenario.

a node. Each node has got limited bandwidth and buffer to transmit the data to other nodes.

\section{B. QoS Metrics}

We propose a mechanism to describe the Quality of connectivity (QoC) for extracting the links connecting the pair of best nodes over time from the network point of view, and use this as the criteria for the route selection algorithm. Reliable network requires more stable nodes and high quality links with better QoC which satisfy bandwidth as a QoS constraint. This is because the performance of ad hoc routing strictly depends on the quality of each individual node. The set of forwarding nodes with higher stability can improve the routing performance. This section presents stability, buffer and bandwidth estimation models used in our routing scheme.

\section{B.1. Node Stability}

The stable nodes are necessary in forwarding group to provide better packet delivery services. Node stability in terms of movement around its current position gives us an idea of stationary property of node. We use node stability metric to identify stable nodes in a path for forwarding packets from a source to the destination.

We identify three metrics to represent node stability as the quality of connectivity from the network point of view: self stability, neighbor nodes stability and buffer level. The steps in finding the stability of a node are as follows. (1) Find the self stability, i.e, when the node is moving to a new position with respect to its previous position. (2) Find neighbors stability of all the nodes in MANET by considering the neighbors self stability, and (3) find the buffer level to indicate the unused portion of the buffer. Each node in the MANET will compute the node stability factor based on these three parameters.

\section{Self Stability}

It can be defined as the node's movement with respect to its previous position. If a node is trying to move away from

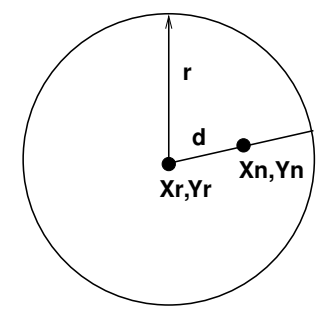

Fig. 2. Node movement.

its position, the distance of the movement and transmission range decides the stability. A node is said to be stable if its movement is within given fraction of its transmission range relative to its previous position.

Consider the scenario shown in Fig. 2, where a node with transmission range ' $\mathrm{r}$ ' moves from position $\left(x_{r}, y_{r}\right)$ to $\left(x_{n}\right.$, $y_{n}$ ) in a given time window ' $\mathrm{t}$ ' by a distance ' $\mathrm{d}$ '.

When a node moves out from its previous position to the next position, its position stability keeps changing with respect to the distance moved. This change in distance $\left(d_{i}^{t}\right)$ of a node ' $i$ ', in a time window ' $t$ ' is estimated by using equation (1).

$$
d_{i}^{t}=\sqrt{\left(x_{n}-x_{r}\right)^{2}+\left(y_{n}-y_{r}\right)^{2}}
$$

Based on the movement of the distance at every time window, the self stability metric $\left(S_{s}(t)\right)$ can be estimated as given in equation (2). $S_{s}(t)$ varies in the range 0 to 1 . When the movement distance $d_{i}^{t}$ of a node increases from its previous position, the self stability value will decrease. For the requirement of the higher degree of movement stability, ' $r / 2$ ' can be replaced by ' $\mathrm{r} / 4$ ' or ' $\mathrm{r} / 8$ '.

$$
S_{s}(t)= \begin{cases}1-\frac{d_{i}^{t}}{r / 2} & \text { if } 0 \leq d_{i}^{t}<r / 2 \\ 0 & \text { Otherwise }\end{cases}
$$

There are some limitations in calculation of self stability due to influence of GPS accuracy and resolution. Better results can be estimated with higher accuracy and resolution GPS, but it requires more cost. GPS has limitation in getting the accurate position information especially when nodes in MANETs are moving with very high speed (more than $20 \mathrm{~m} / \mathrm{sec}$ ). This work assumes that GPS accuracy and resolution is limited to $95 \%$ and 7.8 meters respectively [25].

\section{Neighbor Node Stability}

It can be defined as how well a node is being connected by its neighbor in terms of their self stability. The nodes can exchange messages with each other, if they are within the transmission range. Each node accumulates connectivity information and signal stability of one hop neighbors, and maintains a neighbor list.

The degree of a node ' $n$ ' is represented as number of links (or nodes) connected to it, and is denoted as 'ND'. 
The neighbor node stability of a node $\left(N_{s}(t)\right)$ with respect to neighbors at time ' $\mathrm{t}$ ' can be expressed as in equation (3).

$$
N_{s}(t)=\alpha \times \frac{1}{N D} \sum_{i=1}^{N D} S_{s}^{i}(t)+(1-\alpha) \times N_{s}(t-1)
$$

Where $\alpha$ is the weightage factor (lies between 0 and 1), and is distributed between 0.6 and 0.7 , since they yield better results in simulation. $N_{s}(t-1)$ is the recent neighbor node stability, $S_{s}{ }^{i}(t)$ is the self stability of neighbour node ' $i$ '. We are using the stability model to select nodes with higher self and neighbor stability values such that the selected path through such stable nodes stays for a longer duration.

\section{B.2. Buffer Level}

Stability of a node can also be assessed based on packets/information holding capacity of routing buffer size. If the available buffer level of a particular node is low, then this implies that a large number of packets are lined up for forwarding, which in turn tells that packets routed through this node will have to experience high queuing delays. A high buffer level on the other hand indicates that the corresponding node has few packets queued up for forwarding. Hence a node should maintain the average buffer-level to avoid frequent link failures. Stability of the buffer level is indicated by $B_{S}$, which is defined as the ratio of available buffers to maximum buffer size in a given time window.

\section{B.3. Link Stability}

It indicates the stability of the link between the nodes in terms of quality and life time of the link. The link stability estimated in the scheme is based on two parameters such as, (i) received signal strength, and (ii) life time of the link.

The algorithm 1 represents a pseudocode for updating link stability status between the nodes. The different parameters used in the algorithm are as follows.

- Lifetime: It is the duration of continuous connectivity between the nodes measured in secs;

- lifetime_threshold: It indicates the maximum limit of link lifetime that decides link stability;

- link_stability_status: It is a boolean variable that defines link stability between the nodes;

- Recent: It indicates most recent response received for a Hello packet from a neighbor;

- P: Number of Hello packets;

- received_signal_strength: It is the strength of signal received from a neighbor; and

- signal_threshold: It is an acceptable signal strength to be received from neighbors.

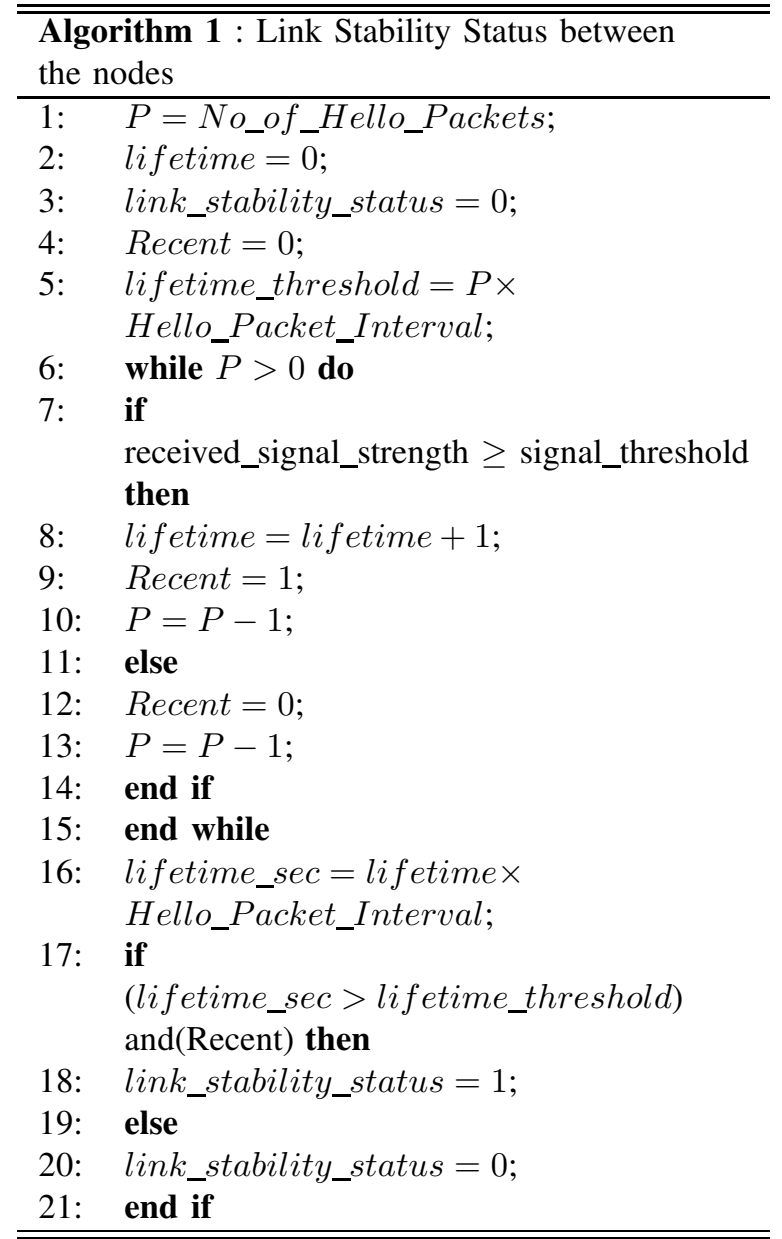

Following parameter values are considered in algorithm 1; signal_threshold $=-8.9 \mathrm{db}$, Hello packet exchange interval $=60 \mathrm{sec}$, No_of_Hello_Packets $=4$, and lifetime_threshold is three times of the Hello packet exchange interval. A typical neighbor information table for a node with neighbors $\mathrm{A}, \mathrm{B}, \mathrm{C}, \ldots$, is given in Tab. I. It comprises of neighbor id and its related information such as Neighbor stability factor, Link stability factor, Recent, lifetime, and link_stability_status. For every neighbor node, link and node stability factor will be estimated as discussed in B.4.

\section{B.4. Stability Factor}

This section describes computation of combined stability factor by using node and link stability factor.

\section{Node Stability Factor}

We need to map the self stability, neighbor nodes stability and buffer level on to a single weighted metric called node stability factor, $N s f$. This can be expressed as in equation (4). The $N s f(t)$ in time interval ' $t$ ' represents the stability of node at the given time interval with respect to its movement and neighbor movement. Higher value of $N s f(t)$ indicates better stability.

$$
\begin{aligned}
& N s f(t)=f\left(S_{s}(t), N_{s}(t), B_{s}(t)\right)= \\
& =\beta S_{s}(t)+\gamma \times N_{s}(t)+\delta \times B_{s}(t)
\end{aligned}
$$

The weight factors $\beta, \gamma$, and $\delta$ denote the relative importance of the quantities $S_{s}(t), N_{s}(t)$, and $B_{s}(t)$, 
TABLE I

NEIGHBOR INFORMATION TABLE

\begin{tabular}{cccccc}
\hline \hline Neighbor id & Neighbor stability factor & Link stability factor & Recent & lifetime(sec) & link_stability_status \\
\hline A & 0.9 & 0.2 & 0 & 3 & 0 \\
B & 0.8 & 0.4 & 1 & 4 & 1 \\
C & 0.6 & 0.3 & 0 & 3 & 0 \\
$\ldots$ & $\ldots$ & $\ldots$ & $\ldots$ & $\ldots$ & $\ldots$ \\
$\ldots$ & $\ldots$ & $\ldots$ & $\ldots$ & $\ldots$ & $\ldots$ \\
\hline \hline
\end{tabular}

respectively. Sum of all the weight factors must be equal to 1. Stability factor of a node is computed only if self stability and neighbor stability is greater than zero. Thus our scheme extracts the highly stable nodes and adjusts the network topology for routing restricted to stable nodes so as to reduce the probability of link failure.

\section{Link Stability Factor}

A node is capable of estimating its neighbor's time of connection called as life time of a node. The node is assumed to be aware of its direct (or immediate) neighbor's relative speed, called as ' $\mathrm{v}$ '. Let us denote the range of a node as ' $r$ ', and the distance moved by the node as $\mathrm{d}$. The remaining distance is (r-d) for which connectivity may still exist. A relationship between these parameters when the link_stability_status = 1 , is given in equation (5), called as link stable duration (Lsd).

$$
L s d=\frac{(r-d)}{v}
$$

Link stable duration can be normalized by using a lifetime_threshold (LTT). This value will not change with respect to time since it is a static value decided by the administrator and its value is relatively larger than each value of the link stable duration (Lsd) in the network scenario. Normalized Lsd, denoted as link stability factor, $L s f$, is given in equation (6).

$$
L s f(t)= \begin{cases}\frac{L s d}{L T T} & \text { if } L s d \leq L T T \\ 1 & \text { Otherwise }\end{cases}
$$

\section{Stability-Factor-Between-Nodes}

Our proposed routing protocol makes use of node stability factor coupled with link stability factor called as StabilityFactor-Between-Nodes (SFBN) for QoS based applications to find the route from a source to destination. SFBN (a normalized value) is as given in equation (7), that helps in selecting stable nodes and links for routing in multihop networks which can stay together for a longer duration.

$$
S F B N(t)=(N s f(t)+L s f(t)) / 2
$$

The path from source to destination will be forwarded through many intermediate links, and the link which is having minimum SFBN will be selected as path SFBN as given in equation (8) denoted by PathSFBN for ' $\mathrm{N}$ ' intermediate links at a given time interval ' $t$ '.

$$
\operatorname{PathSFBN}(t)=\min \left(S F B N_{i}(t)\right) ; \forall i=1 \ldots N
$$

\section{B.5. Bandwidth Estimation}

The bandwidth information is one of the important metric of choice for providing Quality of service (QoS). We considered our previous work presented in [26] to estimate the available bandwidth based on the channel status of the radio link to calculate the idle and busy periods of the shared wireless media. By observing the channel utility, we can take the measure of the activities of the node as well as its surrounding neighbors and thus obtain good approximation of bandwidth usage.

In IEEE 802.11 MANETs, due to the contention based channel access, a node can only transmit data packets after it gains the channel access. Hence a node first listens to the channel and estimates bandwidth on the ratio of idle and busy times for a predefined interval. This can be expressed in the equation form as given in (9).

$$
B W=\frac{T_{\text {idle }}}{T_{\text {interval }}} \times c
$$

Where $T_{\text {idle }}$ denotes the idle time in an interval $T_{\text {interval }}$, and $\mathrm{C}$ denotes the channel capacity. $T_{\text {interval }}$ comprises of the following time periods: idle time of the channel $T_{i d l e}$, time taken for actual transmission of the data $T_{t x}$, time taken for retransmission of packets $T_{r t x}$, and time taken for four-way handshaking $T_{h s}$. Hence equation (9) can be rewritten as in (10).

$$
B W=\frac{T_{i d l e}}{T_{\text {idle }}+T_{t x}+T_{r t x}+T_{h s}} \times c
$$

The above mentioned time periods are measured individually and are incorporated in estimating the bandwidth. Bandwidth in the network is not additive metric, since it is concave in nature. Hence path bandwidth from source to destination will be the minimum of bandwidth of all the links in the path, and is denoted by PathBW for ' $\mathrm{N}$ ' intermediate links in equation (11).

$$
\operatorname{Path} B W=\min \left(B W_{i}\right) ; \forall i=1 \ldots N
$$

\section{Route Establishment}

We have modified Dynamic Source routing (DSR) [27] by applying our QoS metrics and routing scheme. DSR is an on demand (reactive) protocol designed to reduce repeated usage of control packets, hence bandwidth consumption can be reduced, compared to other table driven (proactive) 


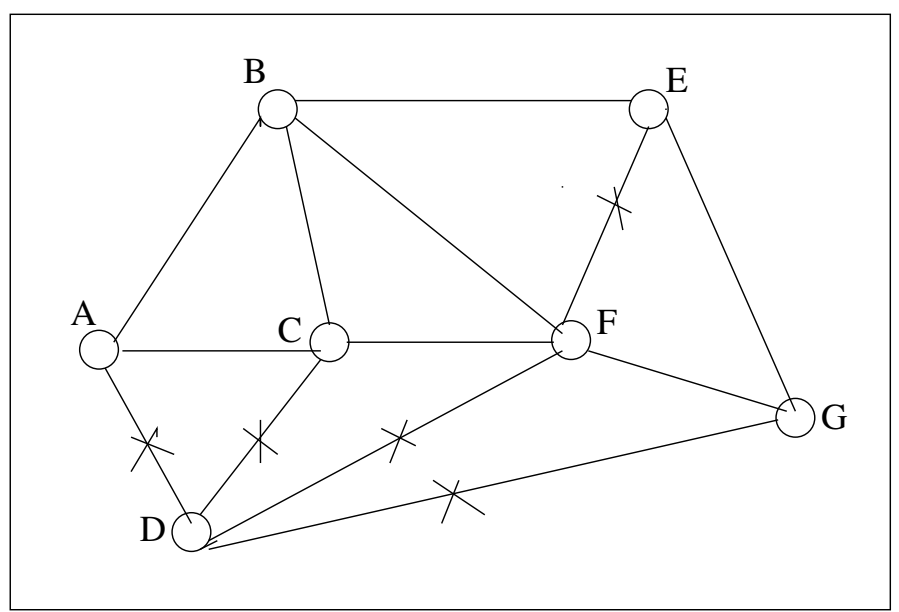

Fig. 3. Network Topology.

protocols. Route establishment makes use of parameters SFBN and bandwidth available information at each node. It considers stability and QoS database at each node for route request propagation and path(s) finding between source to destination. The scheme also uses a routing information cache at each node that facilitates route finding by providing path information; this will reduce route request propagation overheads. This section presents stability and QoS database (NSQB), route request (RR) packets, route reply (RP) packets, route error (RE) packets, and routing information cache (RIC).

\section{C.1. Neighbor Stability and QoS Database}

Each node maintains Neighbor Stability and QoS Database (NSQB) that stores node and link stability related information called as Stability-Factor-Between-Nodes (SFBN) along with bandwidth estimated at each node for satisfying QoS based application in maintaining stable link through intermediate nodes.

To explain the fields of the NSQB we consider the network topology given in Fig 3. Where A, B, C, D, E, F and G are the nodes connected in the network, of which $\mathrm{A}$ is the source node, $\mathrm{G}$ is the destination node and remaining are the intermediate nodes. The crossed links indicate that they are not stable, i.e., SFBN is below the SFTH (stability factor threshold). We consider SFTH to be 0.5 , however the administrator can fix the value between 0.5 and 0.9 to get better quality communication. Table II shows a typical neighbor information table for node A.

The information in the table are: Neighbor id, SFBN and available estimated bandwidth (BW). Neighbor node $\mathrm{D}$ information is not entered in the table since node D's SFBN is below SFTH.

\section{C.2. Route Request, Route Reply and Route Error Packets}

To create a unicast stable QoS route in a MANET from source to destination, various control packets such as route request $(\mathrm{RR})$, route reply (RP) and route error (RE) packets are used. In this section, we describe some of the control packet components required for unicast stable QoS path creation, and
TABLE II

Neighbor Stability and QoS Database (NSQB) at Source Node A

\begin{tabular}{ccc}
\hline \hline Neighbor id & SFBN & BW (Mbps) \\
\hline B & 0.58 & 1.2 \\
C & 0.6 & 1.4 \\
$\ldots$ & $\ldots$ & $\ldots$ \\
$\ldots$ & $\ldots$ & $\ldots$ \\
\hline \hline
\end{tabular}

handling link failure situations. Some important fields of RR packet are as follows.

- Source address: It is the address of the source from where the path need to be established to the destination in the network.

- Destination address: Destination address is the address of the node where packet has to be forwarded. It helps in accommodating the routes created by RR packets and RP packets.

- Time to live: It is the number of hops RR packet can travel. The value is decremented by one at every hop.

- Next hop address: It is the address of the neighbor connected with in the transmission range for propagating RR and RP packet.

- Sequence number: The sequence number assigned to every packet delivered by the source that uniquely identify the packet. It is used to avoid multiple transmission of the same RR packet.

- Route record: It has the addresses of the visited previous nodes recorded in visiting sequence. This information will be used during the return journey to RR packet originator by corresponding RP packet.

- SFBN record: It has the values of SFBN associated with each link which are visited in sequence from the source to destination. This will help in finding path SFBN, which will be used by RP packet to update RIC.

- Available bandwidth record: It is the estimated available bandwidth value associated with each link visited in sequence from source to destination. This will help in finding available path bandwidth, which will be used by RP packet to update RIC.

- Application bandwidth requirement: It is bandwidth required by an application at the source node.

$\mathrm{RP}$ packet format for unicast creation is almost similar to RR packet with few changes in RR packet. The changes in RR packet to convert it into RP packet are as follows: When RR packet reaches the destination, source address and destination address are interchanged, SFBN record will be replaced by one value which is pathSFBN, bandwidth record will be replaced by one value called available path bandwidth, and contents of route record will be reversed. RP packet from the destination is sent to source on a route given in its route record.

RE packet is generated when a node is unable to send the packets. Some of the fields of this packet are source address, destination address, sequence number. Whenever a node identifies link failures, it generates RE packet to either source or destination. If link failure occurs in forward journey of a RR packet (from source to destination), RE packet is sent 
to the source. On the other hand if link failure occurs for reverse journey of the RP packet (from destination to the source), RE packet is sent to the destination. Nodes receiving $\mathrm{RE}$ packet updates their route information cache by removing paths having failed links and also examine its route cache for an alternate path. If an alternate path is found, it modifies the route, otherwise packet is dropped.

\section{C.3. Routing Information Cache (RIC)}

RIC is used to store the latest routes to destinations learned through RR and RP packets. This avoids unnecessary route discovery operation each time when a data packet is to be transmitted. This reduces delay, bandwidth consumption, and route discovery overhead. A single route discovery may yield many routes to the destination, due to intermediate nodes replying from local caches. When source node learns that a route to destination node is broken, it can use another route from its local cache, if such a route to destination exists in its cache. Otherwise, source node initiates route discovery by sending a route request. Use of RIC can speed up route discovery and it can reduce propagation of route requests. The contents of RIC will be removed at every periodic interval, if it is not updated for certain time (may be 180 to 360 seconds).

Each node in the network maintains its own RIC that aids in forwarding packets to neighbors. For every visited RP packet at a node, RIC is updated with by using some of the fields in RP packet required for establishing stable QoS paths. Table III presents a typical RIC at node A for topology given in Fig. 3. Various fields in the table are explained as follows.

- Destination address: It is the address of the node where packet has to be forwarded (extracted from RP packet destination address and route record). It helps in accommodating the routes for RR packets.

- Path information: It represents a complete path (a sequence of links).

- PathSFBN: It is the combined stability factor of path as given in equation 8 .

- PathBW: It is the available path bandwidth which is difference of equation (11) and application bandwidth requirement.

- Recorded timestamp: It contains the time at which RIC is updated by using RP packet.

\section{Route Discovery Process}

Unicast stable QoS path creation involves two phases: a request phase and a reply phase. Request phase invokes route discovery process to find routes to destination using stable and QoS intermediate nodes. Reply phase involves updating of RIC and conforming the routes found in request phase. Stable nodes are the ones which satisfy stability criteria based on our module given in section B as well as accommodate bandwidth requirement of application. These stable and QoS nodes act as intermediate nodes that help to create unicast routes from source to destinations.

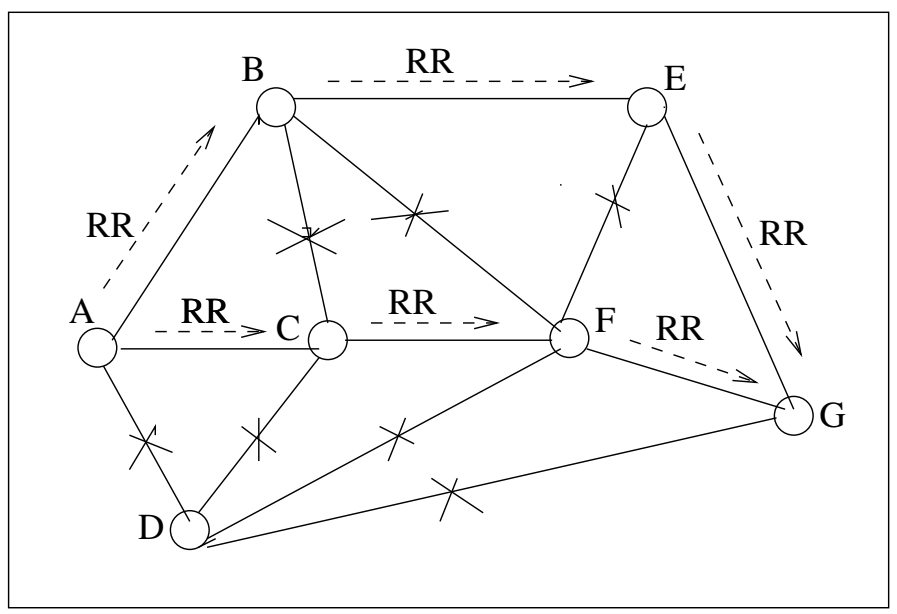

Fig. 4. Route Request Paths from A to G.

In the following section, we present the process of request phase, reply phase, and route maintenance that helps in discovering a path.

\section{D.1. Request Phase}

A source node finds the route to its destination by using RR packets. The sequence of operations that occur are as follows. (1) Source node prepares a RR packet with application bandwidth requirements. (2) Selective transmission of RR packet to neighbors who satisfy stability criteria (i.e., SFBN greater than SFTH) and bandwidth requirements (i.e., estimated bandwidth greater than twice the application requirements). (3) A node receiving $R R$ packet will discard, if it is already received (by using sequence number and source address). (4) If RR packet is not a duplicate, check RIC for availability of route; if available, RP packet will be generated and start reply propagation to source. (5) If RR packet is a duplicate, then discard it and stop transmission of RR packet. (6) If not duplicate and no route available in RIC, transmit the RR packet by updating its fields (route record, SFBN record, bandwidth record, time to live, and nexthop address) to its neighbors as in step 2. (7) Perform steps 3 to 6 until destination is reached, and (8) if destination is not reached within certain hops, send RE packet to the source node.

Figure 4 illustrates the basic operation of route request phase for the network topology of Fig. 3.

- Source node A prepares a RR packet with application bandwidth requirements.

- Send RR packet to neighbors B and C, since they satisfy the SFBN and BW requirement.

- Check for the packet duplication at B and C. Eliminate duplicates.

- Check RIC for availability of route at B and C to G.

- Assuming B and C have no route to G, they update and modify the RR packet (for route record, SFBN record, BW record, Time to live and next-hop add) and transmit to $\mathrm{E}$ and $\mathrm{F}$.

- At E and F, they have no route to $G$, update and modify the RR packet as in previous step, and transmit to $G$. 
TABLE III

Routing Information CACHE (RIC) AT Source Node A

\begin{tabular}{clccc}
\hline \hline Dest Addr & Path information & pathSFBN & Path BW & Recorded Timestamp(H:Min:Sec) \\
\hline G & A-B-E-G & 0.6 & 1.8 & $0: 0: 0.4$ \\
& A-C-F-G & 0.8 & 1.6 & $0: 0: 0.6$ \\
E & A-B-E & 0.7 & 1.0 & $0: 0: 0.8$ \\
$\ldots$ & $\ldots$ & $\ldots$ & $\ldots$ & $\ldots$ \\
$\ldots$ & $\ldots$ & $\ldots$ & $\ldots$ & $\ldots$ \\
\hline \hline
\end{tabular}

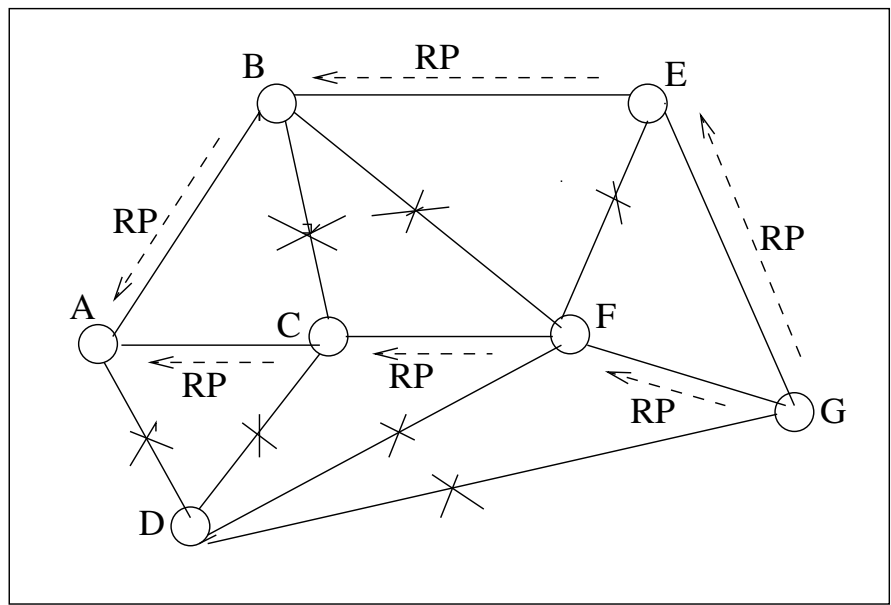

Fig. 5. Route Reply Paths from G to A.

- As $G$ is the destination, and RR packet has reached through two paths A-B-E-G and A-C-F-G; return RP packet to the sender through both the paths.

\section{D.2. Reply Phase}

When RR packet reaches the destination node, following operations are performed in the reply phase. (1) RP packet is generated from RR packet by performing following changes in RR packet; destination and source node addresses are interchanged, route record is reversed, update SFBN record with path SFBN, and update bandwidth record with path bandwidth. (2) Update RIC at destination node with destination id, path information, path SFBN, path bandwidth and time. (3) RP packet is forwarded to next hop node as per the route record. (4) Node receiving RP packet checks whether available bandwidth is greater than application requirement, if so, updates RIC by using contents of RP packet. Updates will happen only if current time is greater than the time recorded in RIC. If bandwidth is not available, send RE packet to destination and visited intermediate nodes and stop RP packet propagation. (5) Perform steps 3 and 4 until source is reached. (6) If source is not found due to link breaks, send RE packet to the destination. (7) The source node chooses one of the received paths with higher bandwidth availability and keeps other paths as backup paths.

Figure 5 illustrates the basic operation of reply phase for the network topology of Fig. 3.

- Node G prepares RP packets for the received RR packets in two directions A-B-E-G and A-C-F-G.
- Route for one RP packet is G-E-B-A and for other RP packet is G-F-C-A. Path SFBN and path bandwidth in the RP packets are updated.

- Both the RP packets are assumed to flow through the paths and reach the source $A$. The visited intermediate nodes will update paths to nodes $\mathrm{G}, \mathrm{E}, \mathrm{B}, \mathrm{C}$, and F in their RIC's.

- RIC at node A will be updated after receiving RP packets in both directions. It updates paths for B. C, E, F, and G.

\section{E. Route Maintenance}

Route maintenance is required in case of link failures. There are three cases of link failures; link failure between stable intermediate nodes, link failure between source and stable intermediate node, and link failure between destination and stable intermediate node. We can tackle the problem in following ways. (1) In case of link failure between two stable intermediate nodes, the node detecting failure condition will use RR and RP packets to find stable QoS path between itself and the destination. The new path from intermediate node to destination will be informed to source. If a new path is not found, the node sends RE packet to source to rediscover the paths. (2) In case of link failure between source and stable intermediate node, source node will probe backup path, if it is working, it will use backup path. Routes will be rediscovered if backup path does not exist. (3) In case of link failure between destination and stable intermediate node, the intermediate node will use RR and RP packets to discover paths to destination from itself and informs the source about the path. If route is not discovered, the node sends RE packet to source to initiate route rediscovery. The source constructs a new path in all the cases for further routing of packets.

\section{Simulation Results}

In this section, we compare the performance of our proposed protocol with RSQR [21], through an extensive set of simulations. Both RSQR and OBSUR are compared interms of traffic admission ratio, packet delivery ratio, control overhead, end to end delay, and throughput. We run the simulation with $95 \%$ confidence interval to analyze the performance parameters.

\section{A. Simulation Model}

We have simulated proposed scheme for various network scenarios using $C$ programming language. Simulation environment for the proposed work consists of four models: (1) Network model, (2) Channel model, (3) Mobility model, and (4) Traffic model. 
Network Model: An ad hoc network is generated in a given area. It consists of several numbers of mobile nodes that are placed randomly within a given area. The coverage area around each node has a limited bandwidth that is shared among it's neighbor. It is assumed that, the operating range of transmitted power and communication range are constant.

Channel Model: It assumes the free space propagation model and error free channel. To access the channel, ad hoc nodes use CSMA/CA (Carrier Sense Multiple Access with Collision Avoidance) media access protocol to avoid possible collisions and subsequent packet drops.

Mobility Model: We use a random way-point (RWP) mobility model based upon three parameters; speed of movement, direction for mobility and time of mobility. In RWP, each node picks a random destination uniformly within an underlying physical space, and travels with a given speed. After reaching the destination, the node pauses for certain time period, and the process repeats itself.

Traffic Model: It is a constant bit rate model that transmits a certain number of fixed size packets in a flow.

\section{B. Performance Metrics}

Following performance metrics have been used to analyze the performance.

- Traffic admission ratio: The ratio between the number of data packets sent to the network from the sources and the number of data packets generated by the sources during the simulation. This metric is closely related to the proportion of established routes in the network based on the policy of each scheme.

- Packet Delivery Ratio (PDR): This is the ratio of the number of data packets received at the destinations and the number of data packets sent by the sources. A high delivery ratio means that both admission control with high reliability for constructing the QoS-aware route and appropriate route maintenance have been conducted.

- Throughput: The amount of data received at the destination during the simulation per unit time. It implies utilization efficiency of bandwidth. Control messages such as the HELLO message, RR, RP and RE are not included in the throughput.

- Control Overhead: This is the ratio of control packets sent to the network and the number of data packets delivered at the destinations.

- End-to-End Delay: This is the delay experienced by the successfully delivered packets in reaching their destinations.

Simulation parameters used are summarized in Tab. IV.

\section{Simulation Procedure}

Simulation procedure for the proposed scheme is as follows:

1) Generate an ad hoc network with the given number of nodes.

2) Estimate stability factor based on self node stability, neighbor node stability and buffer size.
TABLE IV

SIMULATION PARAMETERS

\begin{tabular}{lll}
\hline \hline Sl. No. & Parameter name & Value \\
\hline 1 & Network Area & $\begin{array}{l}1000 \mathrm{~m} \times 1000 \mathrm{~m} \\
\text { flat-grid area }\end{array}$ \\
& & 50 \\
3 & Number of nodes & Random \\
4 & Node placement & Random way-point \\
5 & Mobility model & IEEE 802.11 DCF \\
6 & MAC layer & 2 mbps \\
7 & Channel capacity & 250 \\
8 & Transmission range(m) & 500 \\
9 & Carrier-sense range(m) & Omni directional \\
10 & Antenna type & $0,2,5,10,15,20$ \\
11 & Node speed(m/s) & CBR \\
12 & Traffic type & 512 \\
13 & Packet size(Bytes) & 10 \\
14 & Traffic rate(packets/s) & 40 \\
15 & Minimum bandwidth(Kbps) & 0.1 \\
16 & Maximum delay(s) & 0.5 \\
17 & SFTH(Min) & 0.9 \\
18 & SFTH(Max) & 500 \\
19 & Simulation time(s) & 30 \\
20 & Pause time(s) & $10-15$ \\
\hline \hline
\end{tabular}

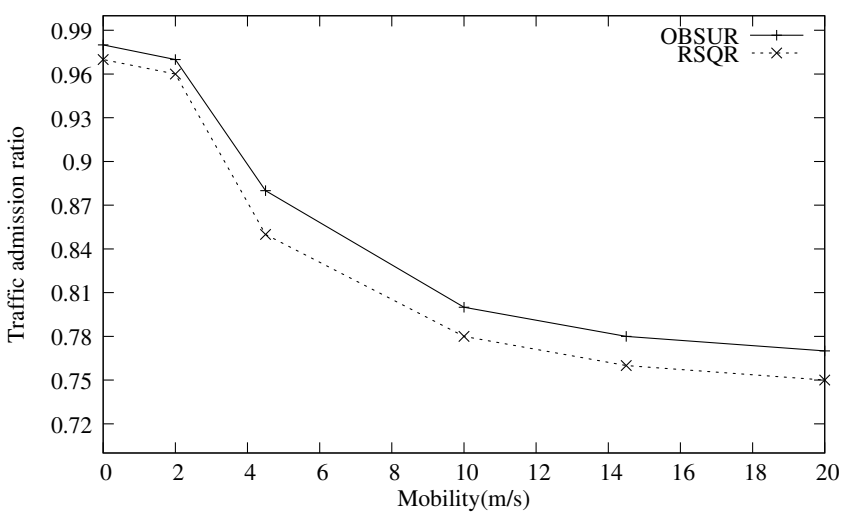

Fig. 6. Traffic admission ratio V/s Mobility(m/s) at low load (10 flows).

3) Compute link stability factor using neighbor information table and Lsd.

4) Compute bandwidth at each node to satisfy application requirement.

5) Update NSQB at each node considering their neighbors.

6) Initiate Route Discovery Process using RR, RP and RE, and accordingly update RIC.

7) Establish the path(s) from source to destination, and send the data packets.

8) Compute performance parameters of the system.

\section{Results}

Figs. 6 and 7 depict traffic admission ratio for 10 and 15 flows, respectively. When the nodes are stationary (i.e., mobility is zero), all traffic generated is accepted and about $98 \%$ of the traffic is able to reach the destination successfully for the 10 flows scenario, whereas it is only about $76 \%$ for the 15 flows. This is because of OBSUR's restrictions on using unstable nodes and weak links. Traffic admission ratio of OBSUR and RSQR at higher mobility (say at 20m/s) 


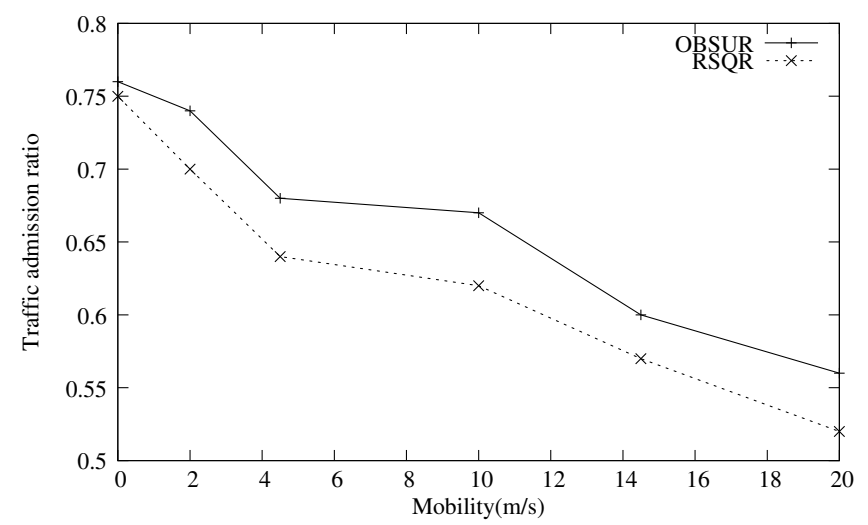

Fig. 7. Traffic admission ratio V/s Mobility $(\mathrm{m} / \mathrm{s})$ at high load (15 flows).

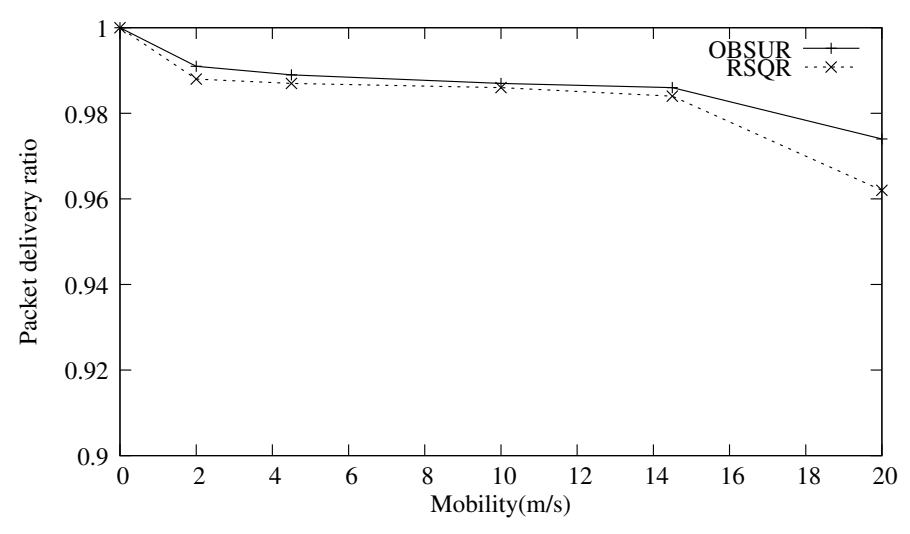

Fig. 8. Packet delivery ratio V/s Mobility(m/s)at low load (10 flows).

are reduced from $78 \%$ and $75 \%$ to $56 \%$ and $53 \%$ for low (10 flows) and high (15 flows) loads, respectively. The admission of connections are low due to node mobility causing unstable links. However, we observe that OBSUR performs better compared to RSQR.

The packet delivery ratio of OBSUR and RSQR is presented in Figs. 8 and 9. For low and high loads, the values are $98 \%$ and $96 \%$, respectively. It is observed that mobility and load variation will not restrict maintaining high values of PDR, which is very much essential to assure high level of QoS. The reasons to achieve high PDR in OBSUR are, (1) use of high stable nodes, high buffer size and longer link life time, (2) reduced control overhead, and (3) maintaining route cache at every node which avoids unnecessary route discovery.

Figs. 10 and 11 represent the normalized control overhead for the increase in mobility for the OBSUR and RSQR protocols. The increase in control overhead at higher mobility is because of frequent link and route failures and also due to QoS violations. As route selection in OBSUR is based upon stable nodes, strong links and higher buffer size, number of route recoveries and hence control overhead encountered are generally less compared to RSQR at low and high mobility. In OBSUR, every node maintains and updates it's route cache frequently, hence usage of control packets is reduced. During low loads, RSQR has $28 \%$ control overhead at $20 \mathrm{~m} / \mathrm{s}$ mobility,

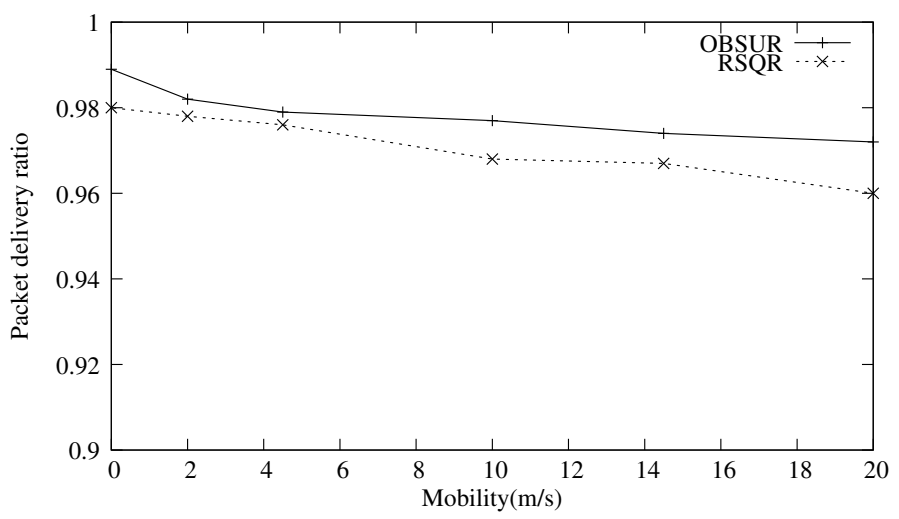

Fig. 9. Packet delivery ratio V/s Mobility(m/s)at high load (15 flows).

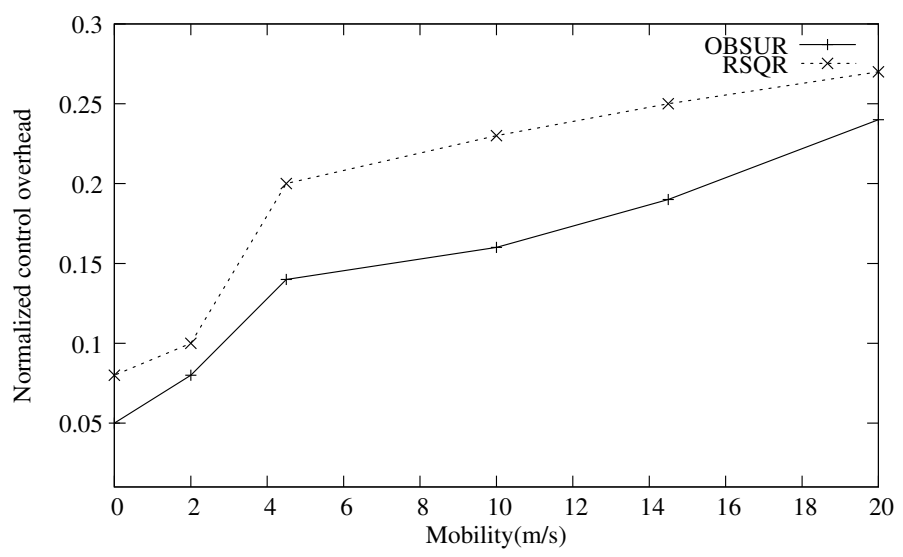

Fig. 10. Normalized control overhead V/s Mobility(m/s)at low load (10 flows).

where as it is only $23 \%$ in OBSUR. The control overhead in OBSUR and RSQR is more at high load condition as shown in Fig. 11. It is $32 \%$ in RSQR and $28 \%$ in OBSUR.

In Figs. 12 and 13, the end-to-end delay decreases with increased mobility. It can be seen that average delay values are $0.013 \mathrm{sec}$ and $0.012 \mathrm{sec}$ for RSQR and OBSUR, respectively at low load, where as these values are $0.022 \mathrm{sec}$ and $0.019 \mathrm{sec}$ at higher loads. This supports the fact that OBSUR has lower delay compared to RSQR.

Throughput measures the effectiveness of a routing protocol in the network. It is the amount of data received at the destination and implies utilization efficiency of bandwidth as the simulation time progresses. OBSUR has the high throughput at minimum size of network as shown in Fig. 14. Control messages such as the HELLO message, RR, RP and RE are not included in the throughput analysis for simplicity. It is observed from Fig. 14 that the throughput value is well above $97 \%$.

\section{CONCLUSIONS}

Bandwidth and stability are the important QoS metrics among several QoS parameters, which are helpful in providing QoS routing for data sensitive and long duration applications. Establishment of a bandwidth and stability constrained path from source to destination in MANETs depends on life time 


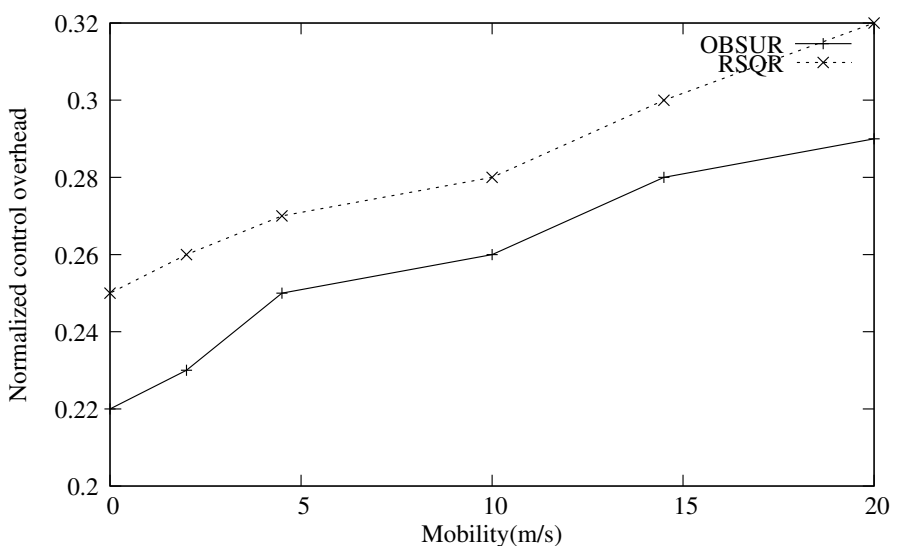

Fig. 11. Normalized control overhead V/s Mobility(m/s)at high load (15 flows).

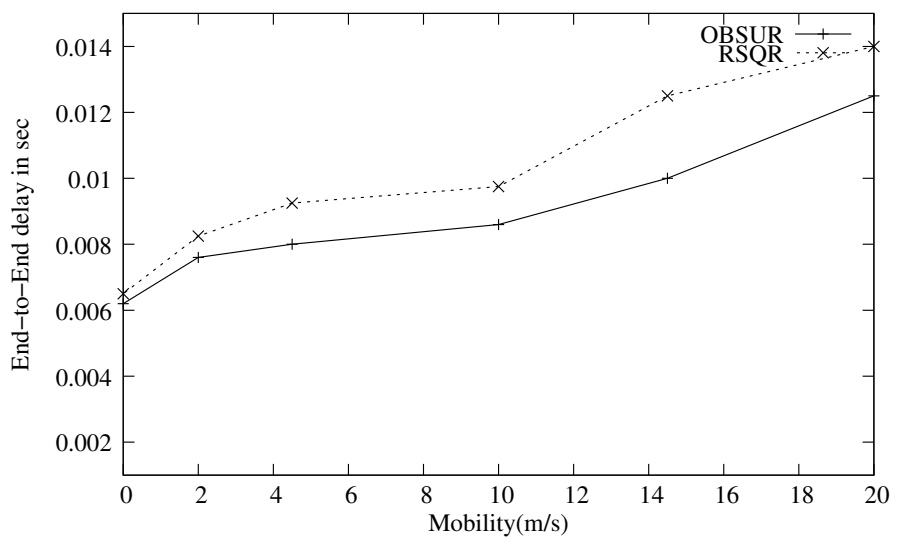

Fig. 12. End-to-End delay in sec. V/s Mobility(m/s)at low load (10 flows).

of the intermediate nodes and link present in the established path and stability of the connected nodes. Node stability factor is computed based on a node movement as well as its neighbor movement with reference to given reference point in a time interval. Link stability factor and buffer levels are also used to support stability factor calculations. In addition to finding the stability factor, a node estimates bandwidth. The selected bandwidth satisfying links going through stable nodes are used for routing the multimedia applications. Simulation results show that there is an improvement in terms of traffic admission ratio, control overhead, packet delivery ratio, end to end delay and throughput as compared to Route Stability Based QoS Routing (RSQR) in MANETs. The work can be extended to find multicast paths for group communication as well as perform cross layer operations with media access layer while routing by using buffer level and link variations.

Practical implementation of the scheme is addressed as follows. The scheme can be implemented on a Linux based mobile nodes by using application programming interfaces (API). The possible implementation may be done as follows. (1) Ad hoc routing scheme can be provided as an API above the kernel space. (2) Bandwidth estimation, stability, and buffer models may be implemented in kernel space and be provided as API's, (3) the data bases can be created in the kernel space except routing data base, which may be

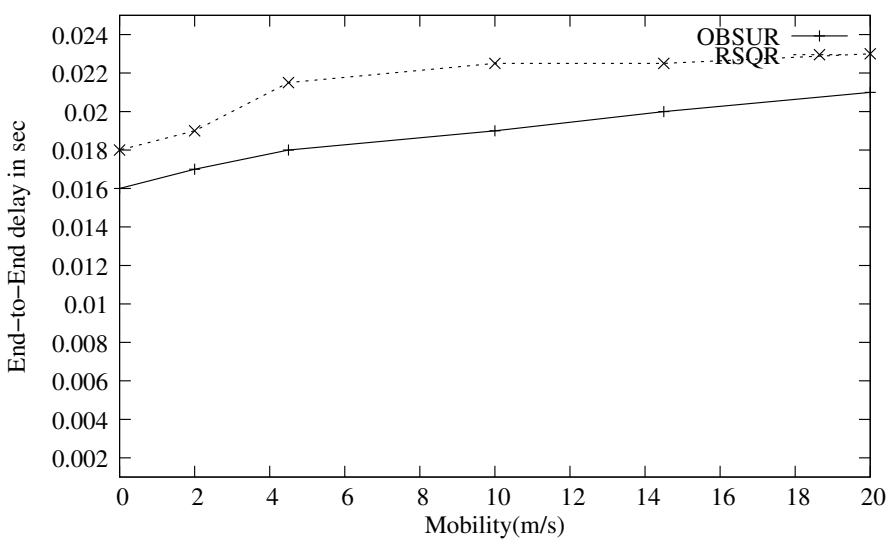

Fig. 13. End-to-End delay in sec. V/s Mobility(m/s)at high load (15 flows).

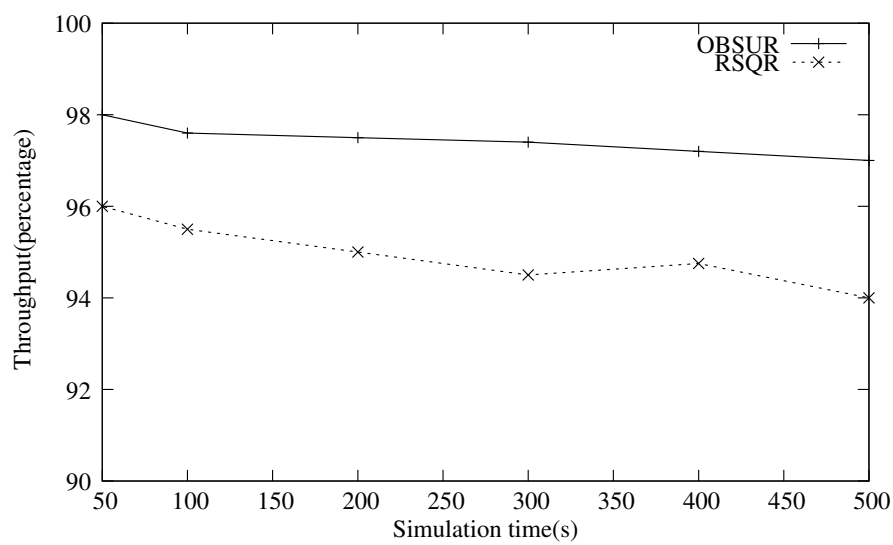

Fig. 14. Throughput in percentage V/s Simulation time(s).

available above kernel space (user space). The software may comprise of user-space library and protocol dependent kernel modules. Routing functionality consists of following parts: parameters estimation (bandwidth, stability, buffers), routing database creation and maintenance, packet forwarding and packet routing.

\section{REFERENCES}

[1] N. Nikaein and C. Bonnet, "Improving routing and network performance in mobile ad hoc networks using quality of nodes," in Proceedings of Wireless Optimization (WiOpt), Sophia-Antipolis, France, 2003.

[2] E. Royer and T. Chai-Keong, "A review of current routing protocols for ad hoc mobile wireless networks," IEEE Personal Communications, vol. 6, no. 2, pp. 46-55, 1999.

[3] J. Augustine, G. Pandurangan, P. Robinson, and E. Upfal, "Towards robust and efficient computation in dynamic peer-to-peer networks," in Proceedings of the Twenty-Third Annual ACM-SIAM Symposium on Discrete Algorithms (SODA '12), Kyoto, Japan, 2012.

[4] R. C. Biradar, S. S. Manvi, and M. Reddy, "Link stability based multicast routing in MANET," Elsevier International Journal on Computer Networks, vol. 54, no. 7, pp. 1183-1196, 2010.

[5] G. Chauhan and S. Nandi, "QoS aware stable path routing (QASR) protocol," in Proceedings of First International Conference on Emerging Trends in Engineering and Technology, Nagpur, India, 2008, pp. 202207.

[6] F. Kuhn, N. Lynch, and R. Oshman, "Distributed computation in dynamic networks," in Proceedings of the 42nd ACM symposium on Theory of computing (STOC-10), Cambridge, Massachusetts, USA, 2010.

[7] G. Carofiglio, C. F. Chiasserini, M. Garettoy, and E. Leonardi, "Route stability in MANETs under the random direction mobility model," IEEE Transaction Mobile Computing, vol. 8, no. 9, pp. 1167-1179, 2009. 
[8] M. R. Brust, C. H. Ribeiro, and S. Rothkugel, "Heuristics on link stability in ad hoc networks," in Proceedings of IEEE Network Operations and Management Symposium (NOMS 2008), Salvador, Bahia, Brazil, 2008, pp. 738-741.

[9] M. R. Brust, S. Rothkugel, and A. Andronache, "Node stability in dynamic communication networks," in Proceedings of the First Asia International Conference on Modeling and Simulation (AMS '07), Phuket, Thailand, 2007, pp. 206-211.

[10] S. Mishra, S. K. Dhurandher, M. Obaidat, N. Nangia, N. Bhardwaj, P. Goyal, and S. Aggarwal, "Node stability-based location updating in mobile ad-hoc networks," IEEE Systems Journal, vol. 2, no. 2, pp. 237 247, 2008.

[11] T. Braun, M. Heissenbuttel, and T. Roth, "Performance of the beacon less routing protocol in realistic scenarios," Elsevier Ad Hoc Networks, vol. 8, no. 1, pp. 96-107, 2010.

[12] H. Wang, Z. Shi, A. Ge, and C. Yu, "An optimized ant colony algorithm based on the gradual changing orientation factor for multi-constraint QoS routing," Elsevier Computer Communications, vol. 32, no. 4, pp. 569-782, 2009.

[13] T. Kunz and R. Alhalimi, "Energy-efficient proactive routing in MANET: Energy metrics accuracy," Elsevier Ad Hoc Networks, vol. 8, no. 7, pp. 755-766, 2010.

[14] P. Sharma, Y. Karkhanawala, and K. Kotecha, "Bandwidth constrained routing of multimedia traffic over hybrid MANETs using ant colony optimization," International Journal of Machine Learning and Computing, vol. 1 , no. 3 , pp. 242-246, 2011.

[15] P. Wannawilai and C. Sathitwiriyawong, "AOMDV with sufficient bandwidth aware," in Proceedings of 10th IEEE International Conference on Computer and Information Technology (CIT 2010), Bradford, West Yorkshire, UK, 2010.

[16] A. Bamis, A. Boukerche, I. Chatzigiannakis, and S. Nikoletseas, "A mobility aware protocol synthesis for efficient routing in ad hoc mobile networks," Elsevier Computer Networks, vol. 52, no. 1, pp. 130-154, 2008.

[17] W. Da-peng, W. Mu-qing, and Z. Yan, "Reliable routing mechanism based on neighbor stability for MANET," Elsevier Computer Networks, vol. 16 , no. 3, pp. 33-39, 2009.
[18] K. A. Yau, P. Komisarczuk, and P. D. Teal, "Reinforcement learning for context awareness and intelligence in wireless networks: Review," Journal of Network and Computer Applications, vol. 35, no. 1, pp. 253 267, 2012.

[19] N. Meghanathan, "A unicast MANET routing protocol to simultaneously minimize the stability-hop count tradeoff and end-to-end Delay," in Proceedings of Ninth International Conference on Information Technology: New Generations (ITNG), Las Vegas, NV, 2012.

[20] R. C. Biradar and S. S. Manvi, "Ring mesh based multicast routing scheme in MANET using bandwidth delay product," Wireless Personal Communications, vol. 66, no. 1, pp. 117-146, 2012.

[21] N. Sarma and S. Nandi, "Route stability based QoS routing in mobile adhoc networks," Wireless Personal Communications, vol. 54, no. 1, pp. 203-224, 2010.

[22] A. Segall, P. Bhagwat, and A. Krishna, "QoS routing using alternate paths," Journal of High Speed Networks - Special issue on quality of service routing, vol. 7, no. 2, pp. 141-158, 1998.

[23] G. Apostolopoulos, R. Guerin, S. Kamat, and S. K. Tripathi, "On reducing the processing cost of on-demand QoS path computation," Journal of High Speed Networks - Special issue on quality of service routing and signaling, vol. 7, no. 2, pp. 77-98, 1998.

[24] A. M. Sukhov and D. Y. Chemodanov, "A metric for dynamic routing based on variational principles," Journal of High Speed Networks, vol. 19, no. 2, pp. 155-163, 2013.

[25] Http://www.gps.gov/systems/gps/performance/accuracy/; Accessed on 15 October, 2012.

[26] P. I. Basarkod and S. S. Manvi, "Multiple parameters based approach to estimate bandwidth in mobile adhoc networks," International Journal of Computer Science Issues, Special Issue (IJCSI), vol. 1, no. 1, pp. 37-43, 2011.

[27] D. B. Johnson and D. A. Maltz, "Dynamic source routing in adhoc wireless networks," Mobile Computing, Kluwer academic publishers, vol. 353, no. 1, pp. 153-181, 1996. 